\title{
Increased blood alpha-carotene, all-trans- Beta-carotene and lycopene levels are associated with beneficial changes in heart rate variability: a CVD-stratified analysis in an adult population-based study
}

Ying Huang ${ }^{1+}$, Hong Chen ${ }^{1 \dagger}$, Yuhao Su${ }^{1}$, Hualong Liu ${ }^{1}$, Jinzhu Hu ${ }^{1 *}$ and Kui Hong ${ }^{1,2^{*}}$

\begin{abstract}
Background: Although the associations of antioxidant micronutrients, such as carotenoids and vitamins, with cardiovascular diseases (CVDs) have been studied extensively, blood concentrations of antioxidant micronutrients and heart rate variability (HRV), which has been proven to be an indicator of cardiac autonomic control, has not been reported. We aimed to explore whether blood concentrations of antioxidant micronutrients, including carotenoids and vitamins, are associated with elevated heart rate variability (HRV (beneficial change) in a crosssectional analysis.
\end{abstract}

Methods: Data were obtained from the Midlife in the United States (MIDUS) study that includes a general adult population. A total of 1074 (aged 34-84) individuals were included. Multivariable analyses were performed to investigate the association between main blood carotenoids (total lutein, zeaxanthin, beta-cryptoxanthin, 13-cis-betacarotene, alpha-carotene, all-trans-beta-carotene and total lycopene) and vitamins A (retinol) and E (gamma-tocopherol and alpha-tocopherol) and HRV after adjustments were made for lifestyle factors and age-related confounders.

Results: Pearson correlation analyses showed that the increased levels of carotenoids and vitamins were positively correlated with higher HRV (all $P<0.05$ ). After adjustments were made for age, gender, race, body mass index(BMI), ever-smoker, number of drinking years and exercise, blood alpha-carotene, all-trans-beta-carotene and total lycopene levels were independently associated with higher HRV in the linear regression model (all $P<0.05$ ). Sensitivity analysis by adding "ever chronic respiratory diseases" as a covariate suggested that blood concentrations of these three carotenoids were still associated with higher low-frequency (LF)-HRV and high-frequency (HF)-HRV (all $P<0.05$ ). Furthermore, stratified analyses suggested that the associations were affected by adding "heart disease" and "hypertension" as covariates.

(Continued on next page)

\footnotetext{
*Correspondence: hujinzhu1983@sina.com; hongkui88@163.com

'Ying Huang and Hong Chen contributed equally to this work.

1 Department of Cardiovascular Medicine, The Second Affiliated Hospital of Nanchang University, No. 1 Minde Road, Donghu District, Nanchang 330006, Jiangxi, China

Full list of author information is available at the end of the article
}

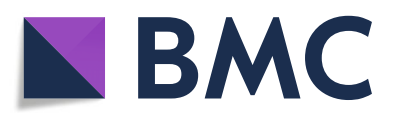

(- The Author(s). 2021 Open Access This article is licensed under a Creative Commons Attribution 4.0 International License, which permits use, sharing, adaptation, distribution and reproduction in any medium or format, as long as you give appropriate credit to the original author(s) and the source, provide a link to the Creative Commons licence, and indicate if changes were made. The images or other third party material in this article are included in the article's Creative Commons licence, unless indicated otherwise in a credit line to the material. If material is not included in the article's Creative Commons licence and your intended use is not permitted by statutory regulation or exceeds the permitted use, you will need to obtain permission directly from the copyright holder. To view a copy of this licence, visit http://creativecommons.org/licenses/by/4.0/. The Creative Commons Public Domain Dedication waiver (http://creativecommons.org/publicdomain/zero/1.0/) applies to the data made available in this article, unless otherwise stated in a credit line to the data. 
(Continued from previous page)

Conclusions: We provide the first evidence that elevated blood concentrations of alpha-carotene, trans-beta-carotene and lycopene are associated with beneficial changes in HRV in the general population. Daily intake of fruit and vegetables may be beneficial to increase blood carotenoid status and further prevent autonomic dysfunction.

Keywords: Carotenoid, Vitamins, Heart rate variability, Cardiovascular diseases, Cross-sectional study

\section{Introduction}

Antioxidant micronutrients, such as carotenoids and vitamins, exist in abundance in fruit and vegetables and have been known to contribute to the body's defense against adverse inflammation and reactive oxygen species $[1,2]$. Numerous epidemiologic studies have demonstrated that a high dietary consumption of fruit and vegetables rich in carotenoids and vitamins with high serum concentrations results in lower risks of cardiovascular diseases (CVDs) [3-6].

CVDs are the leading cause of death and place a high burden of morbidity on the aging populations of industrialized countries [7]. They represent an increasing public health problem because of their high prevalence, need for frequent hospitalization and poor prognosis with detrimental effects at the economic level $[8,9]$. Heart rate variability (HRV) is defined as beat-to-beat fluctuations in heart rate that are mainly determined by the activity of the cardiac sympathetic and parasympathetic nervous systems [10]. It has been widely used as a noninvasive and quantitative marker of cardiac autonomic control. As an indicator of autonomic dysfunction, lower HRV has been associated with a significantly increased risk of myocardial infarction (MI), heart failure (HF), hypertension and CVD death in the general population [11-15]. Furthermore, HRV has also been shown to be related to established risk factors for CVDs in many studies [16-18], suggesting that autonomic dysfunction potentially provides a pathway for CVD-related risk factors to be linked to adverse CVD outcomes.

To our knowledge, few previous studies have comprehensively examined the relationship between the blood levels of the main antioxidants and HRV in general population. The aim of the current study was to examine whether higher carotenoid (i.e., total lutein, zeaxanthin, beta-cryptoxanthin, 13-cis-beta-carotene, alpha-carotene, all-trans-beta-carotene and total lycopene) and vitamin A (retinol) and E (gamma-tocopherol and alphatocopherol) concentrations were associated with beneficial changes in HRV in a community-based, crosssectional study of an adult general population.

\section{Methods}

\section{Study sample}

The data were obtained from 1255 subjects in the MIDUS study, including the behavioral, psychological and social factors in a national sample of adult Americans [19]. Data for the present study are from MIDUS II, a 9-year followup of the MIDUS I cohort, conducted between 2004 and 2006. MIDUS II consisted of a self-administered survey of a wide array of social, behavioral and psychological factors, and a biomarker project and data collection were conducted during a 2-day visit to a clinical research center (CRC) at the University of California-Los Angeles, University of Wisconsin or Georgetown University. Blood data were collected from 2004 to 2009 [20]. Each participant was remunerated $\$ 200$ for participation, and traveling expenses were covered. Clinicians or trained staff evaluated vital signs, morphology, functional capacities and medication usage and performed a physical exam. Medical history was obtained from participants. In summary, a detailed flow chart of the participants included in our study was generated in Fig. 1. After individuals with missing key covariates were excluded, the remaining 1074 individuals were included in the final analysis. In accordance with the Declaration of Helsinki guidelines, the ethics committee of each CRC approved data collection at the three sites, and written consent was obtained from all study participants.

\section{Measurement of blood}

Subjects underwent fasting blood draws prior to breakfast. Samples were sent to the MIDUS Biocore Lab for analysis. The blood concentrations of carotenoids (total lutein, zeaxanthin, beta-cryptoxanthin, 13-cis-beta-carotene, alphacarotene, all-trans-beta-carotene and total lycopene) and vitamins $\mathrm{A}$ and $\mathrm{E}$ were assayed using high-performance liquid chromatography at the Mayo Medical Laboratory (Rochester, MN). The coefficient of variation for the blood test was $6.5 \%$. Participants without a blood carotene measurement $(N=12)$ were excluded. Additionally, glycated hemoglobin, fasting glucose, fasting insulin, total cholesterol, triglycerides, low-density lipoprotein (LDL)-cholesterol and high-density lipoprotein (HDL)-cholesterol assays were analyzed at Meriter Labs (Madison, WI) using a Cobas Integra analyzer (Roche Diagnostics, Indianapolis, IN). CRP and creatinine were measured by a BNII nephelometer (Dade Behring Inc., Deerfield, IL).

\section{Measurement of HRV}

Participants were given with a light breakfast without caffeine after an overnight stay at the CRC. After 


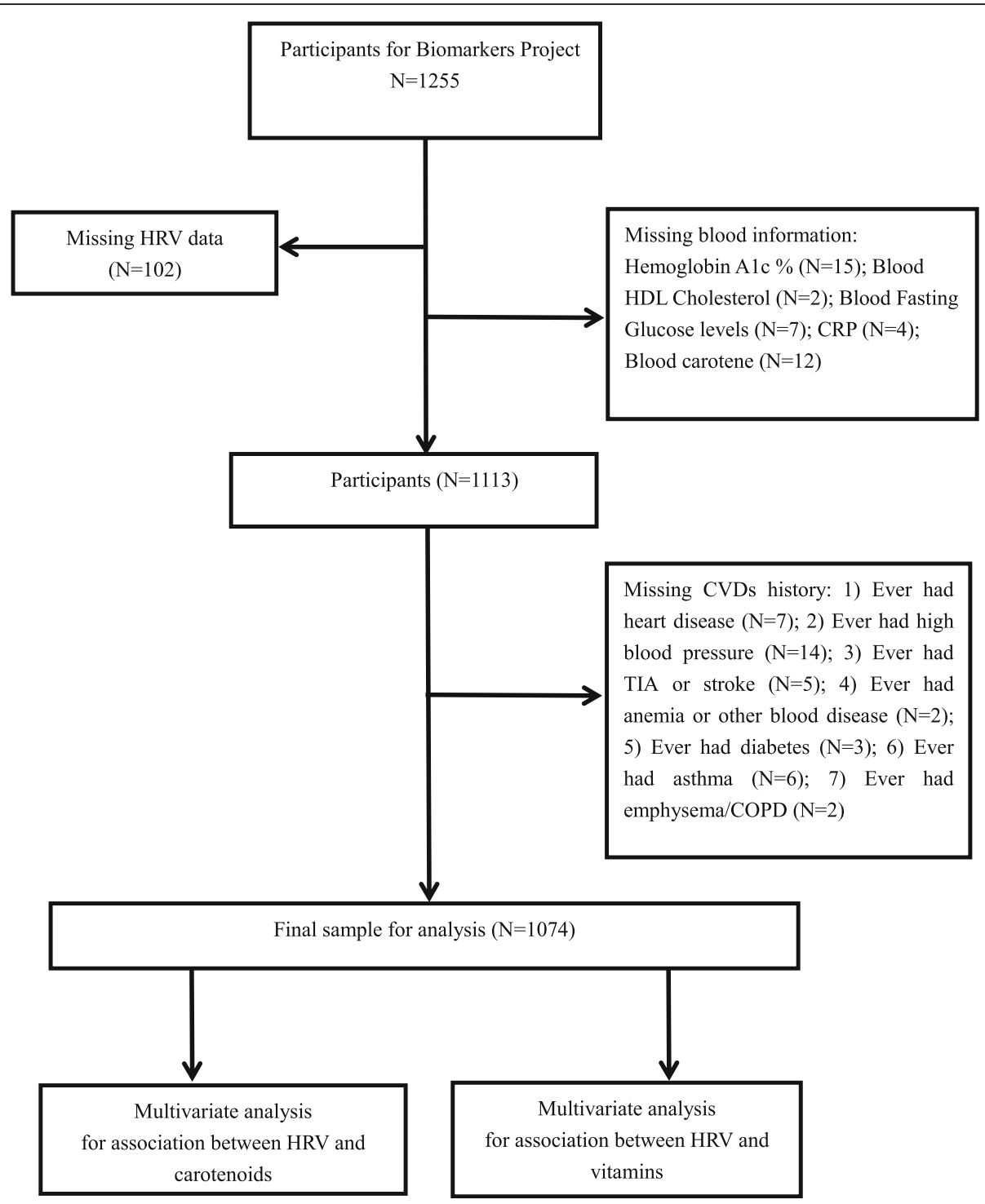

Fig. 1 A detailed flow chart of participants included in the study

breakfast, the psychophysiology protocol of HRV was carried out. ECG electrodes were placed on the right and left shoulders, as well as in the left lower quadrant. Then, respiration bands were placed around the chest and abdomen, and the finger cuff of the beat-to-beat blood pressure monitor was placed around the middle finger on the nondominant hand. The adjustment of respiration was performed by a spirobag of $800 \mathrm{cc}$. Data were recorded during an 11-min baseline as part of a more extensive psychophysiology protocol with exposure to challenging stimuli and recovery periods when participants were in the seated position. Here, we collect HRV data from this resting baseline. Analog ECG signals were digitized at $500 \mathrm{~Hz}$ by a 16-bit A/D conversion board (National Instruments, Austin, TX) and passed to a microcomputer. The ECG waveform was submitted to an R-wave detection routine implemented by customwritten software, resulting in an RR interval series. Errors in marking $\mathrm{R}$ waves were corrected by visual inspection. Ectopic beats were corrected by interpolation. HF-HRV $(0.15-0.40 \mathrm{~Hz})$ was computed based on 300-s epochs using an interval method for computing Fourier transforms similar to a previously described method [21]. The mean value of high-frequency (HF)-HRV from the two baseline 300-s epochs was computed. The process was repeated for low-frequency (LF)HRV $(0.04-0.15 \mathrm{~Hz})$. A higher HRV indicated better cardiac autonomic control.

\section{Covariates}

The results in this study needed to be adjusted for because some variables are known for their associations 
with antioxidant nutrients and HRV. Sociodemographic characteristics and lifestyle factors, including age, sex, race, body mass index (BMI), smoking status, number of drinking years and exercise, were obtained from selfevaluation questionnaires. The questionnaires also collected data on ever having a CVD history, including ever heart disease, ever hypertension, ever transient ischemic attack (TIA) or stroke, ever diabetes mellitus and ever chronic respiratory diseases, including asthma and emphysema/chronic obstructive pulmonary disease (COPD). Race was classified as "white" or "non-white" . Smoking status was also classified as "ever smoker" or "not ever smoker". Exercise was defined as "whether or not has the frequency of exercises $\geq 3$ / week". Selfreported CVD and chronic respiratory disease histories were dichotomized as "yes" or "no".

\section{Statistical analysis}

Analyses in the current study were carried out in SPSS 25.0. The normality of the data was analyzed by the Kolmogorov-Smirnov test combined with Q-Q plots. All continuous variables in the study were not normally distributed, so they were expressed as the median (interquartile range $[\mathrm{IQR}])$. The categoric variables were expressed as $\mathrm{n}(\%)$. Individuals with data missing for a particular variable were removed from our analyses. First, all continuous variables were standardized by using the Z-score and further analyzed by Pearson correlation analysis, which was used to preliminarily evaluate relationships between each of the blood concentrations of 10 antioxidant nutrients and HF and LF-HRV. Multivariate linear regression analysis was further performed to examine the relationships between each antioxidant nutrient and HRV. The significance levels were corrected to account for the 10 associations tested with HF and LF-HRV in each model.

In Model 1, each blood concentration of the 10 antioxidant nutrients was regressed on both HF- and LFHRV with "age" and "gender" adjusted for. Model 2 was adjusted for age, gender, race and BMI. Model 3 was adjusted by adding ever-smoker, number of drinking years and exercise as covariates. Additionally, to further clarify the effect of chronic respiratory diseases (asthma and emphysema/COPD) on blood concentrations of antioxidant nutrients and their relationship and HRV, we added "chronic respiratory diseases" as a covariate for sensitivity analysis. Finally, stratified analyses were performed by adding "heart disease", "hypertension", "TIA or stroke" and "diabetes". Stratification variables were used to examine whether CVDs impacted the association between blood concentrations of antioxidant nutrients and HRV. A $P$ value $\leq 0.05$ was considered statistically significant.

\section{Results}

The characteristics of all the participants

Table 1 presents sociodemographic characteristics, lifestyle factors and medical history for the 1074 participants included in the study. The median age of all participants in this study was 53.0 years, and $43.3 \%$ of them were male. The characteristics of the included participants were as follows: 997 participants (92.8\%) were white; 506 participants (47.1\%) were ever-smokers; and $829(77.2 \%)$ participants had a frequency of exercise $\geq 3$ / week. The median number of drinking years and BMI were 6 and 28.51, respectively. The medians of LF-HRV $(0.04-0.15 \mathrm{~Hz})$ and HF-HRV $(0.15-0.50 \mathrm{~Hz})$ were 233.20 and 126.60 , respectively. The median concentrations of the 10 blood antioxidant nutrients including total lutein, zeaxanthin, beta-cryptoxanthin, 13-cis-beta-carotene, alpha-carotene, all-trans-beta-carotene, total lycopene, gamma-tocopherol, alpha-tocopherol and retinol were $0.229 \mu \mathrm{mol} / \mathrm{L}, 0.055 \mu \mathrm{mol} / \mathrm{L}, 0.163 \mu \mathrm{mol} / \mathrm{L}, 0.054 \mu \mathrm{mol} / \mathrm{L}$, $0.063 \mu \mathrm{mol} / \mathrm{L}, 0.379 \mu \mathrm{mol} / \mathrm{L}, 0.409 \mu \mathrm{mol} / \mathrm{L}, 3.050 \mu \mathrm{mol} / \mathrm{L}$, $25.840 \mu \mathrm{mol} / \mathrm{L}$ and $1.660 \mu \mathrm{mol} / \mathrm{L}$, respectively.

\section{Elevated blood carotenoid levels were positively associated with higher HRV by using Pearson correlation analysis}

Our results with Pearson correlation analyses showed that elevated blood concentrations of carotenoids, including total lutein, zeaxanthin, beta-cryptoxanthin, 13-cis-betacarotene, alpha-carotene, all-trans-beta-carotene, total lycopene and vitamins, including gamma-tocopherol, alpha-tocopherol and retinol, were associated with higher LF-HRV and HF-HRV (all $P<0.05$, Table 2). The single factor correlation analysis preliminarily implied that the blood concentrations of the 10 antioxidant nutrients were closely related to beneficial changes in HRV.

\section{Blood alpha-carotene, all-trans-beta-carotene and total lycopene levels were positively and independently associated with beneficial changes in HRV by using multivariate linear regression analysis}

To further analyze the independent associations of these 10 antioxidant nutrients and HRV, multivariate linear regression analysis was performed (Table 3). Our results indicated that elevated blood concentrations of carotenoids, including total lutein, zeaxanthin, beta-cryptoxanthin, 13cis-beta-carotene, alpha-carotene, all-trans-beta-carotene, total lycopene and vitamins, including gamma-tocopherol, alpha-tocopherol and retinol, were associated with higher LF-HRV and HF-HRV after adjusting for age and gender (all $P<0.05$, Model 1 ). In Model 2, however, only blood alpha-carotene, all-trans-beta-carotene and total lycopene levels were associated with higher LF-HRV and HF-HRV after adjustments were made for age, gender, race and BMI. Model 3 suggested that these three carotenoids were 
Table 1 Characteristics of participants $(N=1074)$

\begin{tabular}{|c|c|}
\hline Variables & $\mathrm{N}(\%)$ or $\mathrm{M}(\mathrm{IQR})$ \\
\hline Age (years) & $53.00(45.00-62.00)$ \\
\hline Gender (male), n (\%) & $465(43.3)$ \\
\hline Race (white), n (\%) & $997(92.8)$ \\
\hline BMI $\left(\mathrm{kg} / \mathrm{m}^{2}\right)$ & $28.51(25.22-32.85)$ \\
\hline Ever smoker, n (\%) & $506(47.1)$ \\
\hline Number of drinking years & $6.00(2.00-23.00)$ \\
\hline Frequency of exercises $\geq 3$ / week, $n$ (\%) & $829(77.2)$ \\
\hline LF-HRV $(0.04-0.15 \mathrm{~Hz})$ & $233.20(104.82-452.88)$ \\
\hline HF-HRV $(0.15-0.50 \mathrm{~Hz})$ & $126.60(56.23-289.83)$ \\
\hline \multicolumn{2}{|l|}{ Ever CVDs history } \\
\hline Heart disease, n (\%) & $99(9.2)$ \\
\hline Hypertension, n (\%) & $380(35.4)$ \\
\hline TIA or stroke, $\mathrm{n}(\%)$ & $37(3.4)$ \\
\hline Diabetes, n (\%) & $132(12.3)$ \\
\hline \multicolumn{2}{|l|}{ Ever chronic respiratory diseases } \\
\hline Asthma, n (\%) & $138(12.8)$ \\
\hline emphysema/COPD, n (\%) & $34(3.2)$ \\
\hline \multicolumn{2}{|l|}{ Blood analysis } \\
\hline Blood Total Lutein (umol/L) & $0.229(0.159-0.339)$ \\
\hline Blood Zeaxanthin (umol/L) & $0.055(0.039-0.083)$ \\
\hline Blood beta-cryptoxanthin (umol/L) & $0.163(0.110-0.254)$ \\
\hline Blood 13-cis-beta-carotene (umol/L) & $0.054(0.035-0.090)$ \\
\hline Blood alpha-carotene (umol/L) & $0.063(0.031-0.115)$ \\
\hline Blood All trans-beta-carotene (umol/L) & $0.379(0.205-0.716)$ \\
\hline Blood Total Lycopene (umol/L) & $0.409(0.298-0.553)$ \\
\hline Blood gamma-tocopherol (umol/L) & $3.050(1.920-4.960)$ \\
\hline Blood alpha-tocopherol (umol/L) & $25.840(20.220-33.530)$ \\
\hline Blood Retinol (umol/L) & $1.660(1.310-2.050)$ \\
\hline Hemoglobin A1c \% & $5.82(5.60-6.20)$ \\
\hline Fasting glucose levels mg/dL & $96.00(90.00-105.00)$ \\
\hline Fasting insulin levels ulU/mL & $10.00(6.00-17.00)$ \\
\hline Total cholesterol (mg/dL) & $184.50(160.00-212.00)$ \\
\hline Triglycerides (mg/dL) & $106.00(77.00-156.25)$ \\
\hline HDL cholesterol (mg/dL) & $53.00(43.00-66.00)$ \\
\hline LDL cholesterol (mg/dL) & $102.00(81.00-128.00)$ \\
\hline Creatinine (mg/dL) & $0.80(0.70-1.00)$ \\
\hline C-reactive protein ( $\mathrm{ug} / \mathrm{mL})$ & $1.39(0.68-3.60)$ \\
\hline
\end{tabular}

$\mathrm{M}$ (IQR) for non-normally distributed variables, and $\mathrm{n}(\%)$ for categoric variables

$B M I$ body mass index; LF-HRV Low frequency-heart rate variability; HF-HRV High frequency heart rate variability; TIA transient ischemic attack; COPD chronic obstructive pulmonary disease; HOMA-IR homeostasis model assessment of insulin resistance; $H D L$ high density lipoprotein; $L D L$ low density lipoprotein
Table 2 Bivariate correlations Using Standardized Variables ( $N=$ 1074)

\begin{tabular}{|c|c|c|c|c|}
\hline \multirow[t]{2}{*}{ Variables } & \multicolumn{2}{|c|}{ LF-HRV } & \multicolumn{2}{|c|}{ HF-HRV } \\
\hline & $\overline{S \beta}$ & $P$ Value & $\overline{S \beta}$ & $P$ Value \\
\hline Blood Total Lutein (umol/L) & 0.068 & 0.016 & 0.068 & 0.016 \\
\hline Blood Zeaxanthin (umol/L) & 0.068 & 0.016 & 0.068 & 0.016 \\
\hline Blood beta-cryptoxanthin (umol/L) & 0.067 & 0.018 & 0.067 & 0.018 \\
\hline Blood 13-cis-beta-carotene (umol/L) & 0.066 & 0.020 & 0.065 & 0.021 \\
\hline Blood alpha-carotene (umol/L) & 0.077 & 0.007 & 0.076 & 0.007 \\
\hline Blood All trans-beta-carotene (umol/L) & 0.111 & $<0.001$ & 0.110 & $<0.001$ \\
\hline Blood Total Lycopene (umol/L) & 0.086 & 0.002 & 0.086 & 0.002 \\
\hline Blood gamma-tocopherol (umol/L) & 0.061 & 0.032 & 0.061 & 0.029 \\
\hline Blood alpha-tocopherol (umol/L) & 0.070 & 0.013 & 0.070 & 0.013 \\
\hline Blood Retinol (umol/L) & 0.068 & 0.016 & 0.068 & 0.016 \\
\hline
\end{tabular}

still significantly related to a higher LF-HRV and HF-HRV after continuing to add ever-smoker, number of drinking years and exercise into Model 2 (all $P<0.05$ ). These results suggested that blood alpha-carotene, all-trans-betacarotene and total lycopene levels are independently associated with beneficial changes in HRV after adjusting for sociodemographic characteristics and lifestyle factors.

Furthermore, the associations between these three carotenoid levels and HRV were determined using sensitivity analysis by adding "respiratory diseases" as a covariate (Table 4), which has been shown to be associated with changed blood levels of antioxidant nutrients $[22,23]$. The results showed that blood alpha-carotene, all-trans-beta-carotene and total lycopene levels were still independently associated with higher LF-HRV and HF-HRV (all $P<0.05$ ). These findings suggested that elevated blood alpha-carotene, all-trans-beta-carotene and total lycopene levels were significantly increased with beneficial changes in HRV.

\section{Stratified analyses of the associations between blood alpha-carotene, all-trans-beta-carotene and total lycopene levels and HRV}

Table 5 presents stratified analyses performed by using CVD history including "ever heart disease", "ever hypertension", "ever TIA or stroke" and "ever diabetes" separately as a stratification variable to assess the associations between blood concentrations of alpha-carotene, all-transbeta-carotene and total lycopene and HRV. Interestingly, in the participants with heart disease and hypertension, we found that the independent associations between blood carotenoid levels (alpha-carotene, all-trans-beta-carotene and total lycopene) and HRV disappeared (all $P>0.05$ ), while the associations of the three blood carotenoid levels with HRV were much stronger in the participants without 
Table 3 Multiple linear regression analysis for relationship between blood carotenoid levels and HRV

\begin{tabular}{|c|c|c|c|c|c|c|}
\hline \multirow[b]{2}{*}{ Variables } & \multicolumn{3}{|c|}{ LF-HRV } & \multicolumn{3}{|c|}{ HF-HRV } \\
\hline & $S \beta$ & $95 \% \mathrm{Cl}$ & $P$ Value & $S \beta$ & $95 \% \mathrm{Cl}$ & $P$ Value \\
\hline \multicolumn{7}{|l|}{ Model 1} \\
\hline Blood Total Lutein & 0.067 & $0.013-0.122$ & 0.016 & 0.067 & $0.012-0.122$ & 0.016 \\
\hline Blood Zeaxanthin & 0.067 & $0.012-0.122$ & 0.016 & 0.067 & $0.012-0.122$ & 0.016 \\
\hline Blood beta-cryptoxanthin & 0.066 & $0.011-0.121$ & 0.018 & 0.066 & $0.011-0.121$ & 0.018 \\
\hline Blood 13-cis-beta-carotene & 0.067 & $0.013-0.122$ & 0.016 & 0.067 & $0.012-0.121$ & 0.017 \\
\hline Blood alpha-carotene & 0.077 & $0.022-0.132$ & 0.006 & 0.076 & $0.022-0.131$ & 0.006 \\
\hline Blood All trans-beta-carotene & 0.114 & $0.060-0.169$ & $<0.001$ & 0.113 & $0.059-0.168$ & $<0.001$ \\
\hline Blood Total Lycopene & 0.085 & $0.031-0.140$ & 0.002 & 0.085 & $0.031-0.140$ & 0.002 \\
\hline lood gamma-tocopherol & 0.064 & $0.010-0.119$ & 0.021 & 0.065 & $0.010-0.120$ & 0.020 \\
\hline Blood alpha-tocopherol & 0.066 & $0.011-0.121$ & 0.018 & 0.066 & $0.011-0.121$ & 0.018 \\
\hline Blood Retinol & 0.066 & $0.011-0.121$ & 0.018 & 0.066 & $0.011-0.120$ & 0.018 \\
\hline \multicolumn{7}{|l|}{ Model 2} \\
\hline Blood Total Lutein & 0.049 & $-0.007-0.104$ & 0.089 & 0.048 & $-0.008-0.104$ & 0.091 \\
\hline Blood Zeaxanthin & 0.048 & $-0.008-0.104$ & 0.090 & 0.048 & $-0.008-0.104$ & 0.092 \\
\hline Blood beta-cryptoxanthin & 0.047 & $-0.008-0.103$ & 0.096 & 0.047 & $-0.009-0.103$ & 0.098 \\
\hline Blood 13-cis-beta-carotene & 0.051 & $-0.004-0.107$ & 0.071 & 0.050 & $-0.005-0.105$ & 0.075 \\
\hline Blood alpha-carotene & 0.060 & $0.005-0.116$ & 0.034 & 0.060 & $0.004-0.115$ & 0.036 \\
\hline Blood All trans-beta-carotene & 0.101 & $0.046-0.156$ & $<0.001$ & 0.100 & $0.045-0.155$ & $<0.001$ \\
\hline Blood Total Lycopene & 0.068 & $0.012-0.124$ & 0.017 & 0.068 & $0.012-0.123$ & 0.017 \\
\hline Blood gamma-tocopherol & 0.045 & $-0.011-0.101$ & 0.112 & 0.046 & $-0.010-0.102$ & 0.106 \\
\hline Blood alpha-tocopherol & 0.047 & $-0.009-0.103$ & 0.098 & 0.047 & $-0.009-0.103$ & 0.099 \\
\hline Blood Retinol & 0.047 & $-0.009-0.103$ & 0.098 & 0.047 & $-0.009-0.103$ & 0.100 \\
\hline \multicolumn{7}{|l|}{ Model 3} \\
\hline Blood Total Lutein & 0.045 & $-0.011-0.101$ & 0.116 & 0.045 & $-0.011-0.101$ & 0.116 \\
\hline Blood Zeaxanthin & 0.045 & $-0.011--0.101$ & 0.118 & 0.044 & $-0.012-0.100$ & 0.121 \\
\hline Blood beta-cryptoxanthin & 0.044 & $-0.012-0.100$ & 0.125 & 0.043 & $-0.013-0.100$ & 0.128 \\
\hline Blood 13-cis-beta-carotene & 0.047 & $-0.008-0.103$ & 0.095 & 0.047 & $-0.009-0.102$ & 0.100 \\
\hline Blood alpha-carotene & 0.057 & $0.002-0.113$ & 0.044 & 0.057 & $0.001-0.112$ & 0.047 \\
\hline Blood All trans-beta-carotene & 0.098 & $0.043-0.153$ & 0.001 & 0.097 & $0.042-0.152$ & 0.001 \\
\hline Blood Total Lycopene & 0.065 & $0.009-0.121$ & 0.023 & 0.064 & $0.009-0.120$ & 0.024 \\
\hline Blood gamma-tocopherol & 0.041 & $-0.015-0.098$ & 0.149 & 0.042 & $-0.014-0.098$ & 0.143 \\
\hline Blood alpha-tocopherol & 0.044 & $-0.012-0.100$ & 0.124 & 0.044 & $-0.012-0.100$ & 0.127 \\
\hline Blood Retinol & 0.044 & $-0.012-0.100$ & 0.126 & 0.043 & $-0.013-0.099$ & 0.130 \\
\hline
\end{tabular}

Model 1: Adjusted for age and gender

Model 2: Adjusted for age, gender, race and BMI

Model 3: Adjusted for age, gender, race, BMI, ever smoker, number of drinking years and exercise

LF-HRV Low frequency-heart rate variability; HF-HRV High frequency heart rate variability

heart disease and hypertension (all $P<0.05$ ). Additionally, in the participants with TIA or stroke and diabetes, however, our results suggested that although the correlation between carotenoid levels and HRV was much weaker in the population with TIA or stroke and diabetes, independent associations between the three carotenoid levels and HRV still existed (all $P<0.05$; Table 5 ). These results suggested that the associations between blood concentrations of alpha-carotene, all-trans-beta-carotene and total lycopene and HRV were affected by heart disease and hypertension, while stroke and diabetes had little effect on associations.

\section{Discussion}

In this community-based, cross-sectional analysis of an adult population, we found that elevated blood alpha- 
Table 4 Sensitivity analysis for relationship between blood carotenoid levels and HRV

\begin{tabular}{|c|c|c|c|c|c|c|}
\hline \multirow[b]{2}{*}{ Variables } & \multicolumn{3}{|c|}{ LF-HRV } & \multicolumn{3}{|c|}{ HF-HRV } \\
\hline & $S \beta$ & $95 \% \mathrm{Cl}$ & $P$ Value & $S \beta$ & $95 \% \mathrm{Cl}$ & $P$ Value \\
\hline \multicolumn{7}{|l|}{ Model 1} \\
\hline Blood alpha-carotene & 0.078 & $0.023-0.132$ & 0.005 & 0.077 & $0.023-0.132$ & 0.006 \\
\hline Blood All trans-beta-carotene & 0.115 & $0.060-0.169$ & $<0.001$ & 0.114 & $0.060-0.168$ & $<0.001$ \\
\hline Blood Total Lycopene & 0.086 & $0.032-0.141$ & 0.002 & 0.086 & $0.031-0.140$ & 0.002 \\
\hline \multicolumn{7}{|l|}{ Model 2} \\
\hline Blood alpha-carotene & 0.061 & $0.005-0.117$ & 0.031 & 0.060 & $0.005-0.116$ & 0.033 \\
\hline Blood All trans-beta-carotene & 0.102 & $0.047-0.157$ & $<0.001$ & 0.101 & $0.046-0.156$ & $<0.001$ \\
\hline Blood Total Lycopene & 0.069 & $0.013-0.124$ & 0.016 & 0.068 & $0.012-0.124$ & 0.016 \\
\hline \multicolumn{7}{|l|}{ Model 3} \\
\hline Blood alpha-carotene & 0.058 & $0.002-0.113$ & 0.042 & 0.057 & $0.001-0.113$ & 0.044 \\
\hline Blood All trans-beta-carotene & 0.098 & $0.043-0.154$ & $<0.001$ & 0.098 & $0.042-0.153$ & 0.001 \\
\hline Blood Total Lycopene & 0.065 & $0.010-0.121$ & 0.022 & 0.065 & $0.009-0.121$ & 0.022 \\
\hline
\end{tabular}

Model 1: Adjusted for age, gender and ever chronic respiratory diseases

Model 2: Adjusted for age, gender, race, BMI and ever chronic respiratory diseases

Model 3: Adjusted for age, gender, race, BMI, ever smoker, number of drinking years, exercise and ever chronic respiratory diseases

LF-HRV Low frequency-heart rate variability; HF-HRV High frequency heart rate variability

carotene, all-trans-beta-carotene and total lycopene levels were strongly associated with beneficial changes in HRV, which may lead to a reduced risk of CVDs through favorable changes in cardiac autonomic function.

The development of autonomic dysfunction in adult population is accompanied by a four-fold higher risk of cardiac autonomic neuropathy, which has been associated with increased CVD mortality [12-14]. HRV has been shown to have direct independent consequences in terms of morbidity and mortality in patients with CVDs, including coronary artery disease, heart failure, stroke and hypertension [11-15]. Although many studies have shown that adequate intake of carotene is beneficial to the prevention of cardiovascular incidence or mortality [24-26], few studies have reported the associations between blood levels of antioxidant micronutrients and HRV that can assess cardiac autonomic function. Previous studies have reported a reduced risk of coronary heart disease, stroke, CVDs and CVD mortality with a high intake of carotenoids [25-33]. Blood concentrations of lycopene were correlated with tomato and tomato juice intake [31,33-35] and the intake of tomatoes has also been inversely associated with coronary heart disease, although associations with other outcomes were less clear [34]. Our results suggested that strong associations between carotenoids and HRV did exist in our results, which was consistent with previous evidence [3-6, 11-15]. We also found that elevated serum total lycopene levels were associated with a beneficial change in HRV. This might be explained by the mechanism by which antioxidant micronutrients contribute to the body's defense against adverse inflammation and reactive oxygen species $[1,2]$, which have been strongly associated with a reduced risk of CVDs through favorable changes in cardiac autonomic function [16-21].

Interestingly, in the participants with heart disease and hypertension, we found that an independent association between blood carotenoid levels (alpha-carotene, alltrans-beta-carotene and total lycopene) and HRV disappeared (all $P>0.05$ ), while these associations were much stronger in the participants without heart disease and hypertension (all $P<0.05$ ). Although there is existing evidence about associations of reduced HRV with an increased risk of CVDs and death [11-15], the results of the stratification differences may be partly explained by the intrinsic impairment in autonomic function caused by coronary heart disease (CAD) or other heart diseases resulting in disruptions in the significant relationships. However, the associations between blood alphacarotene, all-trans-beta-carotene and total lycopene levels and HRV were not affected by diabetes and TIA or stroke. Some studies have suggested that patients with diabetes and TIA or stroke tend to have lower HRV [11-15], a significant association still existed in our study. One possible explanation is that stroke has little effect on autonomic function. Advanced diabetes can cause neuropathy, but early diabetes has little effect on autonomic nerve function. In summary, the differences observed with the stratification of these variables need to be further confirmed in future studies.

Some previous studies have suggested that chronic vitamin $A$ and $E$ administration improved $H R V$ and reduced the risk of CVDs in general populations [36-38]. Potential mechanisms that improve HRV by increasing vitamin intake may reduce the risk of CVDs by 
Table 5 Stratified analysis for association between blood carotenoid levels and HRV by adding "ever CVDs" as covariates

\begin{tabular}{|c|c|c|c|c|c|c|}
\hline \multirow[b]{2}{*}{ Variables } & \multicolumn{3}{|c|}{ LF-HRV } & \multicolumn{3}{|c|}{ HF-HRV } \\
\hline & $S \beta$ & $95 \% \mathrm{Cl}$ & $P$ Value & $S \beta$ & $95 \% \mathrm{Cl}$ & $P$ Value \\
\hline \multicolumn{7}{|l|}{ Heart disease (yes) } \\
\hline Blood alpha-carotene & 0.045 & $-0.019-0.082$ & 0.128 & 0.044 & $-0.020-0.081$ & 0.128 \\
\hline Blood All trans-beta-carotene & 0.049 & $-0.015-0.122$ & 0.099 & 0.048 & $-0.016-0.121$ & 0.100 \\
\hline Blood Total Lycopene & 0.043 & $-0.021-0.100$ & 0.130 & 0.042 & $-0.022-0.099$ & 0.130 \\
\hline \multicolumn{7}{|l|}{ Heart disease (no) } \\
\hline Blood alpha-carotene & 0.069 & $0.004-0.141$ & 0.021 & 0.068 & $0.003-0.140$ & 0.023 \\
\hline Blood All trans-beta-carotene & 0.117 & $0.046-0.173$ & $<0.001$ & 0.116 & $0.045-0.172$ & $<0.001$ \\
\hline Blood Total Lycopene & 0.076 & $0.012-0.128$ & 0.019 & 0.075 & $0.011-0.127$ & 0.019 \\
\hline \multicolumn{7}{|l|}{ Hypertension (yes) } \\
\hline Blood alpha-carotene & 0.047 & $-0.018-0.084$ & 0.122 & 0.046 & $-0.019-0.083$ & 0.122 \\
\hline Blood All trans-beta-carotene & 0.051 & $-0.013-0.125$ & 0.092 & 0.050 & $-0.014-0.124$ & 0.100 \\
\hline Blood Total Lycopene & 0.044 & $-0.020-0.101$ & 0.129 & 0.043 & $-0.021-0.100$ & 0.129 \\
\hline \multicolumn{7}{|l|}{ Hypertension (no) } \\
\hline Blood alpha-carotene & 0.067 & $0.002-0.138$ & 0.025 & 0.066 & $0.001-0.137$ & 0.026 \\
\hline Blood All trans-beta-carotene & 0.114 & $0.044-0.170$ & $<0.001$ & 0.113 & $0.043-0.169$ & $<0.001$ \\
\hline Blood Total Lycopene & 0.075 & $0.011-0.126$ & 0.020 & 0.075 & $0.011-0.126$ & 0.020 \\
\hline \multicolumn{7}{|l|}{ TIA or stroke (yes) } \\
\hline Blood alpha-carotene & 0.057 & $0.001-0.112$ & 0.044 & 0.056 & $0.001-0.112$ & 0.045 \\
\hline Blood All trans-beta-carotene & 0.097 & $0.041-0.153$ & $<0.001$ & 0.096 & $0.040-0.152$ & 0.002 \\
\hline Blood Total Lycopene & 0.064 & $0.0009-0.120$ & 0.023 & 0.064 & $0.008-0.120$ & 0.023 \\
\hline \multicolumn{7}{|l|}{ TIA or stroke (no) } \\
\hline Blood alpha-carotene & 0.059 & $0.002-0.114$ & 0.041 & 0.058 & $0.002-0.113$ & 0.042 \\
\hline Blood All trans-beta-carotene & 0.099 & $0.044-0.156$ & $<0.001$ & 0.099 & $0.043-0.155$ & $<0.001$ \\
\hline Blood Total Lycopene & 0.066 & $0.003-0.122$ & 0.020 & 0.066 & $0.002-0.122$ & 0.020 \\
\hline \multicolumn{7}{|l|}{ Diabetes (yes) } \\
\hline Blood alpha-carotene & 0.056 & $0.001-0.111$ & 0.046 & 0.055 & $0.001-0.110$ & 0.047 \\
\hline Blood All trans-beta-carotene & 0.095 & $0.040-0.151$ & $<0.001$ & 0.094 & $0.039-0.151$ & 0.002 \\
\hline Blood Total Lycopene & 0.063 & $0.008-0.119$ & 0.027 & 0.063 & $0.008-0.118$ & 0.027 \\
\hline \multicolumn{7}{|l|}{ Diabetes (no) } \\
\hline Blood alpha-carotene & 0.060 & $0.004-0.115$ & 0.040 & 0.059 & $0.003-0.114$ & 0.042 \\
\hline Blood All trans-beta-carotene & 0.101 & $0.045-0.157$ & $<0.001$ & 0.100 & $0.044-0.156$ & $<0.001$ \\
\hline Blood Total Lycopene & 0.067 & $0.011-0.126$ & 0.019 & 0.067 & $0.010-0.126$ & 0.019 \\
\hline
\end{tabular}

Adjusted for age, gender, race, BMI, ever smoker, number of drinking years, exercise and ever chronic respiratory diseases

LF-HRV Low frequency-heart rate variability; HF-HRV High frequency heart rate variability

antiarrhythmic, hypotriglyceridemic and antithrombogenic effects, inhibition of atherosclerotic plaque growth and promotion of nitric oxide-induced endothelial relaxation [39]. However, we did not find a significant associations between HRV and blood retinol, alphatocopherol or gamma-tocopherol after adjusting for enough confounding factors, which is inconsistent with previous studies showing that high concentrations of vitamins were associated with a reduced risk of CVDs. The inconsistent results may be at least partly explained by the different study designs used, the different hypotheses being investigated, the different confounding factors included and the inherent difficulties of obtaining epidemiologic measurements of intricate factors. For example, HRV measurements can vary according to psychological, physiological, or environmental factors, especially in individuals across the age range. Additionally, different questionnaires assessing sociodemographic characteristics and lifestyle factors may also contribute to some of these variations.

Important strengths in this study include the community-based design with a large number of 
participants, providing sufficient power to detect small changes in HRV measures. Although not all subjects had repeated measures of HRV, more than one-half of subjects had repeated measures that allowed us to adjust for subject-specific variations in HRV. We treated age, gender, race, BMI, smoking status, drinking status, exercise and other variables as confounding factors, affording better control for them relative to the cross-sectional analysis. Previous studies have only analyzed the relationship between the intake of carotenoids and/or carotenoid-related food and HRV [40]. We collected relevant biochemistry data, including levels of 7 main serum carotenoids, in this study, which is a more intuitive and reliable way to determine the relationship between the blood concentration of carotenoids and HRV. Additionally, given the potential link between carotenoids and CVDs, the associations between blood carotenoids and HRV were further determined using stratified analysis by adding "CVDs" as a covariate. The results still showed that blood alpha-carotene, all-trans-betacarotene and total lycopene levels were independently associated with higher HRV.

This study has several limitations. First, this was a cross-sectional study and it was not possible to make causal inferences between carotenoids and HRV. Second, to participate in this study, all participants needed to be healthy enough to go to a MIDUS study research center. Thus it may lead to potential selection bias. Third, we could not exclude the possibility that the observed associations were attributable to other factors that correlate with blood carotenoids and HRV. Because HRV is a sensitive marker, it can be influenced by some environmental factors. The measurement of serum carotenoid levels is also affected by patients' physiological state, measurement and methods. Fourth, the stratified variables mainly relied on the questionnaire survey provided by the participants, which is not a good method to determine the history of CVDs. This may have caused some deviation in the stratified results. Finally, we could not rule out uncontrolled confounding by other unmeasured variables, and some of our findings may have occurred by chance.

\section{Conclusions}

Our study provides the first evidence that elevated serum alpha-carotene, all-trans-beta-carotene and total lycopene levels are associated with beneficial effects on cardiac autonomic dysfunction in the general population, which may explain why a healthy diet including sufficient carotenoids is an important way to prevent or improve CVDs, even if more research is needed.

\section{Acknowledgments}

We thank all individuals who were responsible for the planning and administration of the MIDUS study and making the datasets of MIDUS available on their website. We also acknowledge the reviewers and editors for viewing our work.

\section{Authors' contributions}

All the authors conceived the study. Ying Huang and Hong Chen contributed to drafting the paper and interpretation of data. Yuhao Su and Hualong Liu reviewed and commented on the manuscript. Jinzhu Hu and Kui Hong reviewed, gave their expert opinion and commented on the manuscript and have given approval of the final version for submission. The author(s) read and approved the final manuscript.

\section{Funding}

This work was supported by grants from the National Natural Science Foundation of China (NSFC 81860070 and 82070350), and JiangXi Province Science Foundation for Distinguished Young Scholar (20202ACBL216001).

\section{Availability of data and materials}

All data generated or analyzed during this study were obtained from MIDUS study.

\section{Declarations}

Ethics approval and consent to participate

Not applicable.

\section{Consent for publication}

Not applicable.

\section{Competing interests}

The authors declared that there are no conflicts of interest.

\section{Author details}

${ }^{1}$ Department of Cardiovascular Medicine, The Second Affiliated Hospital of Nanchang University, No. 1 Minde Road, Donghu District, Nanchang 330006, Jiangxi, China. ${ }^{2}$ Jiangxi Key Laboratory of Molecular Medicine, Nanchang 330006, Jiangxi, China.

Received: 31 December 2020 Accepted: 26 April 2021

Published online: 11 May 2021

\section{References}

1. Margaritelis NV, Paschalis V, Theodorou AA, Kyparos A, Nikolaidis MG. Antioxidants in personalized nutrition and exercise. Adv Nutr. 2018;9(6):81323. https://doi.org/10.1093/advances/nmy052.

2. Wu Q, Liu L, Miron A, Klímová B, Wan D, Kuča K. The antioxidant, immunomodulatory, and anti-inflammatory activities of Spirulina: an overview. Arch Toxicol. 2016;90(8):1817-40. https://doi.org/10.1007/s00204016-1744-5.

3. Isola G, Polizzi A, Alibrandi A, et al. Independent impact of periodontitis and cardiovascular disease on elevated soluble urokinase-type plasminogen activator receptor (suPAR) levels. J Periodontol. 2020. Online ahead of print.

4. Isola G, Polizzi A, Muraglie S, Leonardi R, Lo Giudice A. Assessment of vitamin $C$ and antioxidant profiles in saliva and serum in patients with periodontitis and ischemic heart disease. Nutrients. 2019;11(12):2956. https://doi.org/10.3390/nu11122956.

5. Comstock GW, Bush TL, Helzlsouer K. Serum retinol, betacarotene, vitamin E, and selenium as related to subsequent cancer of specific sites. Am J Epidemiol. 1992;135(2):115-21. https://doi.org/10.1093/oxfordjournals.aje.a116264.

6. D'Odorico A, Martinez D, Kiechl S, et al. High plasma level of alpha and beta-carotene are associated with a lower risk of atherosclerosis. Results from the Bruneck study. Atherosclerosis. 2000;153(1):231-9. https://doi.org/1 0.1016/S0021-9150(00)00403-2.

7. National Institutes of Health NHLaBI. NHLBI fact book fifiscal year 2006.

8. Bursi F, Weston SA, Redfifield MM, Jacobsen SJ, Pakhomov S, Nkomo VT, et al. Systolic and diastolic heart failure in the community. JAMA. 2006; 296(18):2209-16. https://doi.org/10.1001/jama.296.18.2209.

9. Cowie MR, Wood DA, Coats AJ, Thompson SG, Poole-Wilson PA, Suresh V, et al. Incidence and aetiology of heart failure; a population-based study. Eur Heart J. 1999;20(6):421-8. https://doi.org/10.1053/euhj.1998.1280.

10. Task Force of the European Society of Cardiology and the North American Society of Pacing and Electrophysiology. Heart rate variability: standards of 
measurement, physiological interpretation and clinical use. Circulation. 1996; 93:1043-65.

11. Janszky I, Ericson M, Mittleman MA, Wamala S, Al Khalili F, SchenckGustafsson K, et al. Heart rate variability in long-term risk assessment in middle-aged women with coronary heart disease: the Stockholm female coronary risk study. J Intern Med. 2004;255(1):13-21. https://doi.org/10.1046/ j.0954-6820.2003.01250.x.

12. Kearney MT, Fox KA, Lee AJ, Prescott RJ, Shah AM, Batin PD, et al. Predicting death due to progressive heart failure in patients with mild-to-moderate chronic heart failure. J Am Coll Cardiol. 2002;40(10):1801-8. https://doi.org/1 0.1016/S0735-1097(02)02490-7.

13. La Rovere MT, Pinna GD, Maestri R, Mortara A, Capomolla S, Febo O, et al. Short-term heart rate variability strongly predicts sudden cardiac death in chronic heart failure patients. Circulation. 2003;107(4):565-70. https://doi. org/10.1161/01.CIR.0000047275.25795.17.

14. Guzzetti S, La Rovere MT, Pinna GD, Maestri R, Borroni E, Porta A, et al. Different spectral components of $24 \mathrm{~h}$ heart rate variability are related to different modes of death in chronic heart failure. Eur Heart J. 2005;26(4): 357-62. https://doi.org/10.1093/eurheartj/ehi067.

15. Stein PK, Domitrovich PP, Huikuri HV, Kleiger RE. Traditional and nonlinear heart rate variability are each independently associated with mortality after myocardial infarction. J Cardiovasc Electrophysiol. 2005;16(1):13-20. https:// doi.org/10.1046/j.1540-8167.2005.04358.x.

16. Felber Dietrich D, Schindler C, Schwartz J, Barthelemy JC, Tschopp JM, Roche $F$, et al. Heart rate variability in an ageing population and its association with lifestyle and cardiovascular risk factors: results of the SAPA LDIA study. Europace. 2006;8(7):521-9. https://doi.org/10.1093/europace/ eul063.

17. Felber Dietrich D, Schwartz J, Schindler C, Gaspoz JM, Barthelemy JC, Tschopp JM, et al. Effects of passive smoking on heart rate variability, heart rate and blood pressure: an observational study. Int J Epidemiol. 2007;36(4): 834-40. https://doi.org/10.1093/ije/dym031.

18. Hemingway H, Shipley M, Brunner E, Britton A, Malik M, Marmot M. Does autonomic function link social position to coronary risk? The Whitehall II study Circulation. 2005;111(23):3071-7. https://doi.org/10.1161/CIRCULA TIONAHA.104.497347.

19. Brim OG, Ryff CD, Kessler RC. The MIDUS national survey: an overview. In: Brim OG, Ryff CD, Kessler RC, editors. How Healthy Are We? A National 393 Study of Well-Being at Midlife, vol. 392. Chicago: University of Chicago Press; 2004. p. $1-36$

20. Ryff C, Almeida DM, Ayanian JS, Carr DS, Cleary PD, Coe C, et al. National Survey of Midlife Development in the United States (MIDUS II), 2004-2006. ICPSR04652-v6 ed: Inter-university Consortium for Political and Social Research (ICPSR); 2012

21. DeBoer RW, Karemaker JM, Strackee J. Comparing spectra of a series of 395 point events particularly for heart rate variability data. IEEE Trans Biomed Eng. 1984;39631(4):384-7.

22. Day RM, Matus IA, Suzuki YJ, Yeum KJ, Qin J, Park AM, et al. Plasma levels of retinoids, carotenoids and tocopherols in patients with mild obstructive sleep apnoea. Respirology. 2009;14(8):1134-42. https://doi.org/10.1111/j.144 0-1843.2009.01623.x.

23. Kodama Y, Kishimoto Y, Muramatsu Y, Tatebe J, Yamamoto Y, Hirota N, et al. Antioxidant nutrients in plasma of Japanese patients with chronic obstructive pulmonary disease, asthma-COPD overlap syndrome and bronchial asthma[J]. Clin Respir J. 2016;11(6):915-24. https://doi.org/10.1111/ crj.12436.

24. Van Duyn MA, Pivonka E. Overview of the health benefits of fruit and vegetable consumption for the dietetics professional: selected literature. J Am Diet Assoc. 2000;100(12):1511-21. https://doi.org/10.1016/S0002-8223 (00)00420-X.

25. Schwingshackl L, Boeing $H$, Stelmach-Mardas M, Gottschald M, Dietrich S, Hoffmann $G$, et al. Dietary supplements and risk of cause-specific death, cardiovascular disease, and Cancer: a systematic review and meta-analysis of primary prevention trials. Adv Nutr. 2017;8(1):27-39. https://doi.org/10.394 5/an.116.013516.

26. Goyal A, Terry MB, Siegel AB. Serum antioxidant nutrients, vitamin a, and mortality in U.S. adults. Cancer Epidemiol Biomark Prev. 2013;22(12):2202-11. https://doi.org/10.1158/1055-9965.EPI-13-0381.

27. Aune D, Keum N, Giovannucci E, Fadnes LT, Boffetta P, Greenwood DC, et al. Dietary intake and blood concentrations of antioxidants and the risk of cardiovascular disease, total cancer, and all-cause mortality: a systematic review and dose-response meta-analysis of prospective studies. Am J Clin Nutr. 2018;108(5):1069-91. https://doi.org/10.1093/ajcn/nqy097.

28. Huang J, Weinstein SJ, Yu K, Männistö S, Albanes D. Serum Beta carotene and overall and cause-specific mortality. Circ Res. 2018;123(12):1339-49. https://doi.org/10.1161/CIRCRESAHA.118.313409.

29. Huang J, Weinstein SJ, Yu K, Männistö S, Albanes D. Relationship between serum alpha-Tocopherol and overall and cause-specific mortality. Circ Res. 2019;125(1):29-40. https://doi.org/10.1161/CIRCRESAHA.119.314944.

30. Cheng HM, Koutsidis G, Lodge JK, Ashor AW, Siervo M, Lara J. Lycopene and tomato and risk of cardiovascular diseases: a systematic review and meta-analysis of epidemiological evidence. Crit Rev Food Sci Nutr. 2019; 59(1):141-58. https://doi.org/10.1080/10408398.2017.1362630.

31. Hak AE, Stampfer MJ, Campos H, Sesso HD, Gaziano JM, Willett W, et al. Plasma carotenoids and tocopherols and risk of myocardial infarction in a low-risk population of US male physicians. Circulation. 2003;108(7):802-7. https://doi.org/10.1161/01.CIR.0000084546.82738.89.

32. Maillard V, Kuriki K, Lefebvre B, Boutron-Ruault MC, Lenoir GM, Joulin V, et al. Serum carotenoid, tocopherol and retinol concentrations and breast cancer risk in the E3N-EPIC study. Int J Cancer. 2010;127(5):1188-96. https:// doi.org/10.1002/ijc.25138.

33. Hendrickson SJ, Willett WC, Rosner BA, Eliassen AH. Food predictors of plasma carotenoids. Nutrients. 2013;5(10):4051-66. https://doi.org/10.3390/ nu5104051.

34. Aune D, Giovannucci E, Boffetta P, Fadnes L, Keum N, Norat T, et al. Fruit and vegetable intake and the risk of cardiovascular disease, total cancer and all-cause mortality - a systematic review and dose-response meta-analysis of prospective studies. Int J Epidemiol. 2017:46(3):1029-56. https://doi.org/10.1 093/ije/dyw319.

35. Tyssandier V, Feillet-Coudray C, Caris-Veyrat C, Guilland JC, Coudray C, Bureau $S$, et al. Effect of tomato product consumption on the plasma status of antioxidant microconstituents and on the plasma total antioxidant capacity in healthy subjects. J Am Coll Nutr. 2004;23(2):148-56. https://doi. org/10.1080/07315724.2004.10719355.

36. Varela-López A, Navarro-Hortal MD, Giampieri F, Bullón P, Battino M, Quiles U. Nutraceuticals in periodontal health: a systematic review on the role of vitamins in periodontal health maintenance. Molecules. 2018;23(5):1226. https://doi.org/10.3390/molecules23051226.

37. Murai U, Yamagishi K, Sata M, Kokubo Y, Saito I, Yatsuya H, et al. Seaweed intake and risk of cardiovascular disease: the Japan public health centerbased prospective (JPHC) study. Am J Clin Nutr. 2019;110(6):1449-55. https://doi.org/10.1093/ajcn/nqz231.

38. Galan P, Kesse-Guyot E, Czernichow S, Briancon S, Blacher J, Hercberg S, et al. Effects of $B$ vitamins and omega 3 fatty acids on cardiovascular diseases: a randomised placebo controlled trial. BMJ. 2010 Nov 29;341: c6273.

39. Galan P, Viteri FE, Bertrais S, Czernichow S, Faure H, Arnaud J, et al. Serum concentrations of betacarotene, vitamins $C$ and $E$, zinc and selenium are influenced by sex,age, diet, smoking status, alcohol consumption and corpulence in a general French adult population. Eur J Clin Nutr. 2005; 59(10):1181-90. https://doi.org/10.1038/sj.ejcn.1602230.

40. Park SK, Tucker KL, O'Neill MS, Sparrow D, Vokonas PS, Hu H, et al. Fruit, vegetable, and fish consumption and heart rate variability: the veterans administration normative aging study. Am J Clin Nutr. 2009;89(3):778-86. https://doi.org/10.3945/ajcn.2008.26849.

\section{Publisher's Note}

Springer Nature remains neutral with regard to jurisdictional claims in published maps and institutional affiliations. 